\title{
K-COMPONENT DISCONJUGACY FOR SYSTEMS OF ORDINARY DIFFERENTIAL EQUATIONS
}

\author{
JOHNNY HENDERSON \\ Department of Mathematics \\ Auburn University \\ Auburn, Alabama 36849
}

(Received October 3, 1985)

ABSTKACT: Disconfugacy of the $k$ th component of the $m$ th order system of $n$th order differential equations $Y^{(n)}=f\left(x, Y, Y^{\prime}, \ldots, Y^{(n-1)}\right),(1.1), 1$ s defined, where $f\left(x, Y_{1}, \ldots, Y_{n}\right), \frac{\partial f}{\partial y_{1 f}}\left(x, Y_{1}, \ldots, Y_{n}\right):(a, b) \times R^{m n} \rightarrow R^{m}$ are continuous. Given a a solution $Y_{0}(x)$ of $(1.1)$, k-component disconfugacy of the variational equation $Z^{(n)}=\prod_{1=1}^{n} f_{Y_{1}}\left(x, Y_{0}(x), \ldots, Y_{0}^{(n-1)}(x)\right) Z^{(1-1)},(1.2)$, 1s also studied. Conditions are given for continuous dependence and differentiablitty of solutions of (1.1) w1th respect to boundary conditions, and then intervals on which (1.1) 1s k-component disconjugate are characterized in terms of intervals on which (1.2) 1s k-component is confugate.

KEY WORDS AND PHRASES. System of differential equations, varlational equation, $k$-component disconjugacy ( $r$ ight disfocal1ty), continulty and differentiabllity with respect to boundary conditions.

1980 AMS SUBJECT CLASSIFICATION: 34B10, 34B15.

1. INTRODUCTION.

In the past several years, a number of results have been proven concerning the disconjugacy of an $n$th order scalar ordinary differential equation when certain disconjugacy assumptions are made for the corresponding linear varlational equation. In this paper we investigate simflar concepts for systems of ordinary differential equations. In particular, we shall be concerned with solutions of boundary value problems for the $m$ th order system of $n$th order differential equations

$$
Y^{(n)}=f\left(x, Y, Y^{\prime}, \ldots, Y^{(n-1)}\right) \text {, }
$$

where we assume throughout:

(A) $f\left(x, Y_{1}, \ldots, Y_{n}\right):(a, b) \times R^{m n} \rightarrow R^{m}$ is continuous;

(B) $\frac{\partial \mathrm{f}}{\partial y_{1 f}}\left(x, Y_{1}, \ldots, Y_{n}\right):(a, b) \times R^{m n}+R^{m}, 1 \leq f \leq m, 1 \leq 1 \leq n$, are continuous, 
where $Y_{1}=\left(y_{11}, \ldots, y_{1 m}\right)$; (Note: If $Y=\left(y_{1}, \ldots, y_{m}\right) \varepsilon R^{m}$, then $Y_{\hat{k}}$ will w111 denote the $(m-1)$-tuple $\left.\left(y_{1}, \ldots, y_{k-1}, y_{k+1}, \ldots, y_{m}\right)\right)$

(C) Solutions of ( 1$)$ extend to $(a, b)$;

and (D) If there exist a sequence of solutions $\left\{Y_{r}(x)\right\}$ of (1.1), a point $\mathrm{x}_{0} \in(\mathrm{a}, \mathrm{b})$ a compact subinterval $[\mathrm{c}, \mathrm{d}] \subset(\mathrm{a}, \mathrm{b}), \mathrm{M}>0$, and $1 \leq \mathrm{k} \leq \mathrm{m}$ such that $\left(Y_{\mu}\right)_{\hat{k}}^{(1-1)}\left(x_{0}\right)=\left(Y_{\nu}\right)_{\hat{k}}^{(1-1)}\left(x_{0}\right), 1 \leq 1 \leq n$, for all $\mu, \nu \in N$, and $\left|\bar{y}_{\mu k}(x)\right| \leq M$ on $\{c, d]$, for all $\mu \varepsilon N$, then there is a subsequence $\left\{Y_{r_{j}}(x)\right\}$ such that $\left\{y_{r_{j}}^{(1-1)}(x)\right\}$ converges uniformly on each compact subinterval of $(a, b)$, for $1 \leq 1 \leq n$. Given a solution $Y_{0}(x)$ of $(1.1)$, we will also be concerned with solutions of the linear $m$ th order system of $n$th order equations called the variational equation along $Y_{0}(x)$ and given by

$$
z^{(n)}=\sum_{1=1}^{n} f_{Y_{1}}\left(x, Y_{0}(x), \quad Y_{0}^{\prime}(x), \ldots, Y_{0}^{(n-1)}(x)\right) z^{(1-1)},
$$

where $f_{\mathbf{r}_{1}}, 1 \leq 1 \leq \mathrm{n}$, denotes the $m \times m$ Jacobian matrix $\left[\frac{\partial f_{k}}{\partial y_{1 j}}\right], 1 \leq k, j \leq m$. Rather than with the disconjugacy of $(1.1)$, we will be concerned with the ilsconjugacy of one of the components of the system (1.1). Motivation for our considerations here are papers by Peterson [1-2], Spencer [3], and Sukup [4]. DEPINITION. Let $1 \leq k \leq m$ be given. We say that (1.1) 1s $\frac{k \text {-component }}{<}$ disconjugate on $(a, b)$, if given $2 \leq q \leq n$, points $a<x_{1}<\ldots<x_{q}<b$, $x \in(a, b)$, positive integers $m_{1}, \ldots, m_{q}$ partitioning $n$, and solutions $Y(x)$ and $Z(x)$ of (1.1) satisfying

$$
\mathbf{Y}_{\hat{k}}^{(1-1)}(\alpha)=z_{\hat{k}}^{(1-1)}(\alpha), 1 \leq 1 \leq n,
$$

and

$$
y_{k}^{(1)}\left(x_{f}\right)=z_{k}^{(1)}\left(x_{f}\right), 0 \leq 1 \leq m_{f}-1,1 \leq f \leq q,
$$

it follows that $y_{k}(x) \equiv z_{k}(x)$.

Given a solution $Y_{0}(x)$ of $(1.1)$, k-component disconjugacy of $(1,2)$ along $\mathrm{Y}_{0}(\mathrm{x})$ is defined similarly.

In this paper, we first show that if the system (1.1) is k-component disconjugate, for some $1 \leq k \leq m$, then solutions of certaln boundary value problems for (1.1) can be differentlated with respect to boundary conditions. The resulting partial derivatives as functions of $x$ are solutions of related boundary value problems for the system (1.2). The main results of this paper appear in section 3 , where we show that intervals on which (1.1) is k-component disconfugate can be characterized in terms of intervals on which (1.2) 1s k-component disconfugate. Then In our last section, we state without proof some analogues of the results in section 3 in terms of k-component right disfocallty for the system (1.1).

2. CONTINUITY AND DIPPERENTIABILITY WITH RESPECT TO BOUNDARY CONDITIONS. Our first result is concerned with the continuous dependence of solutions of (1.1) on boundary conditions. Its proof is a standard application of the Brouwer Invarlance of Domain Theorem. 
THEOREM 1. Assume that for some $1 \leq k \leq m$, the system (1.1) 18 $k$-component disconfugate on $(a, b)$. Let $Y(x)$ be a solution of $(1.1)$. Given $2 \leq q \leq n$, points $a<x_{1}<\ldots<x_{q}<b, \alpha \varepsilon(a, b)$, positive integers $m_{1}, \ldots, m_{q}$ partitioning $n$, and $\varepsilon>0$, there exists $\delta>0$ such that $\left|t_{f}-x_{j}\right|<\delta, 1 \leq f \leq q, 1 \nabla_{\hat{k}}^{(1-1)}(\alpha)-$ $\left(\nabla_{1}\right)_{\hat{k}} 1<\delta, 1 \leq 1 \leq n,\left|y_{k}^{(1)}\left(x_{j}\right)-c_{1 j}\right|<\delta, 0 \leq 1 \leq m_{j}-1,1 \leq j \leq q, 1 m p 1 y$ there exists a unique solution $z_{\delta}(x)$ of $(1.1)$ satisfying $\left(z_{\delta}\right)_{\hat{k}}^{(1-1)}(\alpha)=$ $\left(v_{1}\right)_{\hat{k}}, 1 \leq 1 \leq \mathrm{n}, z_{\delta k}^{(1)}\left(t_{f}\right)=c_{1 f}, 0 \leq 1 \leq m_{f}-1,1 \leq 1 \leq q$, and $\lim _{j \rightarrow 0^{+}} Z_{\delta}^{(1)}(x)=Y^{(1)}(x)$ unfformly on each compact subinterval of $(a, b)$, for $0 \leq 1 \leq n-1$.

In addition to the continuous dependence in Theorem 1, connectedness properties have played an important role in establishing disconfugacy or disfocallty results in the papers of Henderson [5], Peterson [2], and Sukup [4].

THEOREM 2. Assume that for some $1 \leq k \leq m$, the system (1.1) 1s $k$-component ilsconfugate on $(a, b)$. Let $Y(x)$ be a solution of $(1.1)$ and let $4, x_{1}, \ldots, x_{q}, a$, and $m_{1}, \ldots, m_{q}$ be as in Theorem 1 . Then, for $1 \leq p \leq q$, the set $S_{p}=\left\{v_{k}^{\left(m_{p}-1\right)}\left(x_{p}\right) \mid v(x)\right.$ is a solution of $(1.1), \nabla_{\hat{k}}^{(1-1)}(\alpha)=$ $\mathbf{z}_{\hat{k}}^{(1-1)}(\alpha), 1 \leq 1 \leq n, v_{k}^{(1)}\left(x_{f}\right)=y_{k}^{(1)}\left(x_{f}\right), 0 \leq 1 \leq m_{j}-1,1 \leq j \leq q, j \neq p$, and $\left.v_{k}^{(1)}\left(x_{p}\right)=y_{k}^{(1)}\left(x_{p}\right), 0 \leq 1 \leq m_{p}-2\right\} \quad$ is an open interval.

PROOP. It follows Immediately from Theorem 1 that $S_{p}$ is open. It suffices to show that if $\left.\tau_{0}=\sup \left\{\tau \mid\left(y_{k}{ }^{(m}-1\right)\left(x_{p}\right), \tau\right] \subset S_{p}\right\}$ and $\tau^{\prime}>\tau_{0}$, then $\tau^{\prime} \notin S_{p}$, and If $\sigma_{0}=\operatorname{lnf}\left\{\sigma \mid\left[\sigma, y_{k}{ }^{\left(m_{p}-1\right)}\left(x_{p}\right)\right] \subset s_{p}\right\}$ and $\sigma^{\prime}<\sigma_{0}$, then $\sigma^{\prime} \& s_{p}$. We w111 make the the argument for the first case. We suppose that there exists $\tau^{\prime}>\tau_{0}$ and $\tau^{\prime} \varepsilon S_{p}$ Then there is a solution $V(x)$ of (1.1) satisfying $\nabla_{\hat{k}}^{(1-1)}(\alpha)=x_{\hat{k}}^{(1-1)}(\alpha), 1 \leq 1 \leq n, v_{k}^{(1)}\left(x_{f}\right)-\nabla_{k}^{(1)}\left(x_{j}\right), 0 \leq 1 \leq m_{j}-1$, $1 \leq f \leq q, f \neq p, v_{k}^{(1)}\left(x_{p}\right)=y_{k}^{(1)}\left(x_{p}\right), 0 \leq 1 \leq m_{p}-2$, and $v_{k}^{\left(m_{p}-1\right)}\left(x_{p}\right)=\tau^{\prime}$. Now, from the definition of $\tau_{0}$, there exists a strictly monotone increasing sequence $\left\{\tau_{\mu}\right\} \subset S_{p}$ such that $y_{k}{ }^{\left(m_{p}-1\right)}\left(x_{p}\right)<\tau_{\mu}<\tau_{0}$ and $\tau_{\mu}+\tau_{0}$. Let $\left\{u_{\mu}\right\}$ be the corresponding sequence of solutions (1.1) satisfying $\left(w_{\mu}\right)_{\hat{k}}^{(1-1)}(\alpha)=y_{k}^{(1-1)}(\alpha), 1 \leq 1 \leq n, w_{\mu k}^{(1)}\left(x_{j}\right)=y_{k}^{(1)}\left(x_{j}\right), 0 \leq 1 \leq m_{j}-1,1 \leq f \leq q$, $f \neq p, w_{\mu k}^{(1)}\left(x_{p}\right)=y_{k}^{(1)}\left(x_{p}\right), 0 \leq 1 \leq m_{p}-2$, and $w_{\mu k}^{\left(m_{p}-1\right)}\left(x_{p}\right)=\tau_{\mu}$. Now if for some $>0,\left\{w_{\mu k}^{\left(m_{p}-1\right)}(x)\right\}$ is uniformly bounded on $\left[x_{p}, x_{p}+\varepsilon\right]$, it follows from the boundary conditions that $\left\{w_{\mu k}(x)\right\} \quad 18$ unlformly bounded on $\left[x_{p}, x_{p}+\varepsilon\right]$. By condition (D), there exists a subsequence $\left\{u_{\mu_{j}}(x)\right\}$ such that $\left\{w_{\mu j}^{(1-1)}(x)\right\}$ converges uniformly on compact subintervals of $(a, b)$, for $1 \leq 1 \leq n$. In particular, this convergence is uniform on any compact sublnterval containing $a$, and consequently, the subsequence $\left\{u_{\mu_{f}}(x)\right\}$ converges uniformly to a solution $u_{0}(x)$ of $(1.1)$ on compact subintervals of $(a, b)$. Thus, it follows that ${ }^{\tau} 0 \varepsilon S_{p}$, which is 
contradictory to the fact that $S_{p}$ is open. Hence, given $\left.\varepsilon>0, w_{\mu k}^{\left(m_{p}\right.}{ }^{-1)}(x)\right\}$ is not uniformly bounded on $\left[x_{p}, x_{p}+\varepsilon\right]$.

ince, for each $\mu \in N, y_{k}^{\left(m_{p}-1\right)}\left(x_{p}\right)<w_{\mu k}^{\left(m_{p}-1\right)}\left(x_{p}\right)<v_{k}{ }^{\left(m_{p}-1\right)}\left(x_{p}\right)$, 1t follows that there exists a sequence $\left\{\delta_{f}\right\}$ with $\delta_{f}+0$ such that, elther

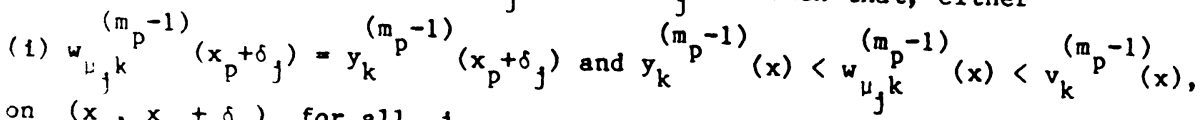
on $\left(x_{p}, x_{p}+\delta_{f}\right)$, for all $f$, or (11) $w_{\mu_{f} k}^{\left(m_{p}-1\right)}\left(x_{p}+\delta_{f}\right)=v_{k}^{\left(m_{p}-1\right)}\left(x_{p}+\delta_{f}\right)$ and $y_{k}^{\left(m_{p}-1\right)}(x)<w_{\mu_{j} k}^{\left(m_{p}-1\right)}(x)<$ $v_{k}{ }^{\left(m_{p}-1\right)}(x)$, on $\left(x_{p}, x_{p}+\delta_{f}\right)$, for all $f$. We then have by continulty that $\lim _{f \rightarrow \infty} w_{\mu j}^{(1)}\left(x_{p}+\delta_{f}\right)=y_{k}^{(1)}\left(x_{p}\right), 0 \leq 1 \leq m_{p}-2$, and hence by Theorem $1,\left\{w_{\mu_{f}}^{(1)}(x)\right\}$ converges to $y_{k}^{(1)}(x)$ uniformly on each compact subinterval of $(a, b)$, for $0 \leq 1 \leq n-1$; a contradiction. This completes the proof.

Dur next result deals with differentiation of solutions of (1.1) with respect to boundary conditions in the presence of $k$-component disconjugacy. The proof follows along the lines of those given in Henderson [5-6] and Peterson [1] and we w111 omit it here.

THEOREM 3. Let $1 \leq k \leq m$ be given and assume that (1.1) and the varlational equation (1.2) along all solutions $I(x)$ of (1.1) is k-component disconjugate on $(a, b)$. Let $Y(x)$ be a solution of $(1.1)$, and let $2 \leq q \leq a$, polnts $a<x_{1}<\ldots<x_{q}<b, a \varepsilon(a, b)$, and positive integers $m_{1}, \ldots, m_{q}$ partitioning $n$ be given. For $1 \leq p \leq q$, let $s_{p}$ be as in Theorem 2 , and for each $s \in S_{p}$, let $\nabla(x, s)$ denote the corresponding solution of $(1.1)$ where $v_{k}\left(m_{p}-1\right)\left(x_{p}, 8\right)=8$. Then $\frac{\partial V}{\partial s}(x, s)$ exists and $z(x, s) \equiv \frac{\partial V}{\partial s}(x, s)$ 1s the solution of $(1.2)$ along $\nabla(x, 8)^{28}$ and satisfles the boundary conditions

$$
\begin{aligned}
& \mathbf{z}_{\hat{k}}(1-1)(\alpha)=0,1 \leq 1 \leq \mathrm{n}, \\
& z_{k}^{(1)}\left(x_{f}\right)=0,0 \leq 1 \leq m_{f}-1,1 \leq f \leq q, j \neq p, \\
& z_{k}^{1}\left(x_{p}\right)=0,0 \leq 1 \leq m_{p}-2, \\
& z_{k} p^{-1)}\left(x_{p}\right)=1 \text {. }
\end{aligned}
$$

3. INTERVALS OF K-COMPONENT DISCONJUGACY.

In this section, we determine subintervals of $(a, b)$ on which (1.1) 1s $k$-component disconfugate in terms of sublatervals on which (1.2) is $k$-component disconjugate. Our arguments for this characterization are much 11ke those in Peterson [2] and Spencer [3].

Por notational purposes, given $\alpha \varepsilon(a, b)$, let $Y\left(x ; \alpha, v_{1}, \ldots, v_{n}\right)$ denote the solution of the Inftial value problem for $(1.1)$ satisfying $\mathbf{Y}^{(1-1)}(\alpha)=v_{1}=$ $\left(v_{11}, \ldots, v_{1 m}\right), 1 \leq 1 \leq n$. Then, under our assumptions (A) - (D), for each 
$1 \leq \mu \leq n$ and $1 \leq \nu \leq m, v_{\mu \nu}\left(x ; \alpha, v_{1}, \ldots, \nabla_{n}\right) \equiv \frac{\partial}{\partial v_{\mu \nu}}\left(x ; \alpha, v_{1}, \ldots, \nabla_{n}\right)$ exists and ts a solution of $(1.2)$ along $Y\left(x ; \alpha, v_{1}, \ldots, \nabla_{n}\right)$ satisfying $\sigma_{\mu \nu}^{(1-1)}(\alpha)=0$, $=0,1 \leq 1 \leq n, 1 \neq \mu, \quad \sigma_{\mu \nu}^{(\mu-1)}(\alpha)=e_{\nu}=\left(\delta_{1 \nu}, \ldots, \delta_{m \nu}\right)$.

DEFINITIONS. Let $1 \leq k \leq m$ be given and let $t \varepsilon(a, b)$.

(1) Define $\eta_{1}^{k}(t)=\operatorname{lnf}\left\{t_{1} \varepsilon(t, b) \mid\right.$ (1.1) is not k-component disconjugate on $\left.\left\{t, t_{1}\right]\right\}$. If $(1.1)$ is $k$-component disconjugate on $(t, b)$, we set $n_{1}^{k}(t)=b$.

(11) Given a solution $Y_{0}(x)$ of $(1.1)$, define $n_{1}^{k}\left(t ; Y_{0}(x)\right)=\operatorname{lnf}\{t \varepsilon(t, b) \mid$

(1.2) is not k-component disconjugate along $Y_{0}(x)$ on $\left.\left[t, t_{1}\right]\right\}$.

The main result of this paper is that $\eta_{1}^{k}(t)=\operatorname{lnf}_{Y_{0}(x)}\left\{\eta_{1}^{k}\left(t ; Y_{0}(x)\right)\right\}$ which w111 be established in two parts. Similar to the argument in Spencer [3], we first prove that $\left.n_{1}^{k}(t) \leq \operatorname{lnf}_{Y_{0}}(x)=n_{1}^{k}\left(t ; Y_{0}(x)\right)\right\}$. The proof of the final theorem of the section shows that strict inequality is not possible, hence the equality will be established. THEOREM 4. Let $1 \leq k \leq m$ be given. Then $\eta_{1}^{k}(t) \leq \operatorname{lnf}_{\mathbf{Y}_{0}(x)}\left\{\eta_{1}^{k}\left(t ; \mathbf{P}_{0}(x)\right)\right\}$. PROOP. Let $\tau=\operatorname{lnf}_{Y_{0}(x)}\left\{n_{1}^{k}\left(t ; Y_{0}(x)\right)\right\}$ and let $\varepsilon>0$ be given. Then on the interval $[t, \tau+\varepsilon)$, there exist $2 \leq q \leq n$, points $t \leq x_{1}<\ldots<x_{q}<\tau+\varepsilon$, $a \varepsilon[t, \tau+\varepsilon)$, positive integers $m_{1}, \ldots, m_{q}$ partitioning $n$, and a non-trivial solution $Z\left(x ; Y_{0}(x)\right)$ of the variational equation $(1.2)$ along a solution $Y_{0}(x)$ of $(1.1)$, such that $z_{k}^{(1-1)}\left(\alpha ; Y_{0}(x)\right)=0,1 \leq 1 \leq n$, and $z_{k}^{(1)}\left(x_{f} ; Y_{0}(x)\right)=0$, $0 \leq 1 \leq \mathrm{m}_{\mathrm{f}}-1,1 \leq \mathrm{f} \leq \mathrm{q}$.

By disconjugacy arguments similar to those in Henderson [7], Muldowney [8], and Peterson [9], it follows that there is a solution $W\left(x ; Y_{0}(x)\right)$ of (1.2) along $Y_{0}(x)$ and points $t \leq t_{1}<\ldots<t_{n}<\tau+\varepsilon$ such that $w_{\hat{k}}^{(1-1)}\left(\alpha ; Y_{0}(x)\right)=0$, $1 \leq 1 \leq n, w_{k}\left(x ; Y_{0}(x)\right)$ has a simple zero at $x=t_{1}, 1 \leq 1 \leq n-1$, and has an odd order zero at $x=t_{n}$. Now for sultable constants $C_{1 k}, 1 \leq 1 \leq n, W\left(x ; Y_{0}(x)\right)=$ $i_{t=1}^{n} C_{1 k} \sigma_{1 k}\left(x ; \alpha, \nabla_{1}, \ldots, \nabla_{n}\right)$, where $r_{0}^{(1-1)}(\alpha)=v_{1}, 1 \leq 1 \leq n$. For $h \neq 0$, consider now the difference quotient

$\left.\frac{1}{h} Y\left(x ; \alpha,\left(v_{1}\right)_{\hat{k}}, v_{1 k}+h C_{1 k}, \ldots,\left(\nabla_{n}\right)_{\hat{k}}, v_{n k}+h C_{n k}\right)-Y\left(x ; \alpha, \nabla_{1}, \ldots, \nabla_{n}\right)\right\}=$

$\frac{1}{h}\left[\begin{array}{c}y_{1}\left(x ; \alpha,\left(v_{1}\right)_{\hat{k}}, v_{1 k}+h c_{1 k}, \ldots,\left(v_{n}\right)_{\hat{k}}, v_{n k}+h c_{n k}\right)-y_{1}\left(x ; \alpha, v_{1}, \ldots, v_{n}\right) \\ y_{m}\left(x ; \alpha,\left(v_{1}\right)_{k}, v_{1 k}+h c_{1 k}, \ldots,\left(v_{n}\right)_{\hat{k}}, v_{n k}+h c_{n k}\right)-y_{m}\left(x ; \alpha, v_{1}, \ldots, v_{n}\right)\end{array}\right]$.

By adding and subtracting, to the $f$ th component, $1 \leq f \leq m$, of the quotient, terms of the form $y_{j}\left(x ; \alpha, v_{1}, \ldots,\left(v_{s}\right) \hat{k}, v_{s k}+h c_{s k}, \ldots\right.$, $\left.\left(v_{n}\right)_{k}, v_{n k}+h C_{n k}\right)$, we obtain 
$\frac{1}{h}\left\{Y\left(x ; \alpha,\left(v_{1}\right)_{\hat{k}}, v_{1 k}+h C_{1 k}, \ldots,\left(\nabla_{n}\right)_{\hat{k}}, v_{n k}+h C_{n k}\right)-Y\left(x ; \alpha, v_{1}, \ldots, \nabla_{n}\right)\right\}=$

$C_{1 k}\left[\begin{array}{c}u_{1 k l}\left(x ; \alpha,\left(\nabla_{1}\right)_{\hat{k}}, v_{1 k}+\xi_{1 k l},\left(\nabla_{2}\right)_{k}, v_{2 k}+h C_{2 k}, \ldots,\left(v_{n}\right)_{\hat{k}}, v_{n k}+h C_{n k}\right) \\ \vdots \\ u_{1 k m}\left(x ; \alpha,\left(\nabla_{1}\right)_{\hat{k}}, v_{1 k}+\xi_{1 k m},\left(\nabla_{2}\right)_{k}, v_{2 k}+h C_{2 k}, \ldots,\left(\nabla_{n}\right)_{\hat{k}}, v_{n k}+h C_{n k}\right)\end{array}\right]$

$+\ldots+$

$C_{n k}\left[\begin{array}{c}u_{n k 1}\left(x ; \alpha, v_{1}, \ldots, v_{n-1},\left(\nabla_{n}\right)_{k}, v_{n k}+\xi_{n k 1}\right) \\ \vdots \\ u_{n k m}\left(x ; \alpha, \nabla_{1}, \ldots, \nabla_{n-1},\left(\nabla_{n}\right)_{\hat{k}}, v_{n k}+\xi_{n k m}\right)\end{array}\right]$, where for each

$1 \leq \nu \leq m, \xi_{\mu k \nu}$ is between 0 and $h C_{\mu k}$. Hence, as $h+0$, the difference quotient (3.1) converges uniformly on compact subintervals to

$c_{1 k}\left[\begin{array}{c}u_{1 k 1}\left(x, \alpha, v_{1}, \ldots, v_{n}\right) \\ u_{1 k m}\left(x ; \alpha, v_{1}, \ldots, v_{n}\right)\end{array}\right]+\ldots+c_{n k}\left[\begin{array}{c}u_{n k 1}\left(x, \alpha, v_{1}, \ldots, v_{n}\right) \\ \vdots \\ u_{n k m}\left(x ; \alpha, v_{1}, \ldots, v_{n}\right)\end{array}\right]=$

$\Gamma_{1=1}^{n} c_{1 k} U_{1 k}\left(x ; \alpha, v_{1}, \ldots, v_{n}\right)$. Thus, for $h$ sufficiently small, the

difference $P(x) \equiv Y\left(x ; \alpha,\left(\nabla_{1}\right)_{\hat{k}}, v_{1 k}+h C_{1 k}, \ldots,(\nabla)_{\hat{k}}, v_{n k}+h C_{n k}\right)$

- $Y\left(x ; \alpha, \nabla_{1}, \ldots, \nabla_{n}\right)$ satisfies the conditions $P_{\hat{k}}^{(1-1)}(\alpha)=0,1 \leq 1 \leq n$,

and $p_{k}\left(\sigma_{1}\right)=0,1 \leq 1 \leq n$, for some $t \leq \sigma_{1}<\sigma_{2}<\ldots<\sigma_{n}<\tau+\varepsilon$.

It follows that $\eta_{1}^{k}(t)<\tau+\varepsilon$, and since $\varepsilon>0$ was arbitrary, we have

$\eta_{1}^{k}(t) \leq \operatorname{lnf}_{\mathbf{Y}_{0}(x)}\left\{\eta_{1}^{k}\left(t ; Y_{0}(x)\right)\right\}$.

THEOREM 5. Let $1 \leq k \leq m$ be given. Then $n_{1}^{k}(t)=\operatorname{lnf}_{0}(x)\left\{n_{1}^{k}\left(t ; Y_{0}(x)\right\}\right.$.

PROOF. L,et $\sigma=\operatorname{lnf}_{\mathbf{Y}_{0}(x)}\left\{\eta_{1}^{k}\left(t ; Y_{0}(x)\right)\right\}$ and assume that $\eta_{1}^{k}(t)<\sigma$. On

the set $\left\{\left(m_{1}, \ldots, m_{q}\right)\right\}$, where $m_{1}, \ldots, m_{q}$ are positive integers partitioning $n, 1 \leq q \leq n$, we define a lexicographical ordering by $\left(n_{1}, \ldots, n_{q}\right)>\left(m_{1}, \ldots, m_{p}\right)$, If $n_{1}>m_{1}$, or If there ex1sts $s \in\{1, \ldots, q-1\}$ such that $n_{1}=m_{1}, 1 \leq 1 \leq 8$, and $\mathrm{n}_{8+1}>\mathrm{m}_{8+1}$.

Since we are assuming that $\eta_{1}^{k}(t)<\sigma$, there exist a last tuple $\left(m_{1}, \ldots, m_{q}\right)$, points $t \leq x_{1}<\ldots<x_{q}<\sigma, \alpha \varepsilon[t, \sigma)$, and distinct solutions $Y(x)$ and $W(x)$ of

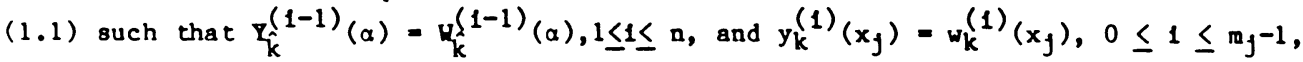
$1 \leq f \leq q . \quad\left(m_{1}, \ldots, m_{q}\right)$ is the last tuple for such distinct solutions, hence $y_{k}^{\left(m_{1}\right)}\left(x_{1}\right) \neq w_{k}^{\left(m_{1}\right)}\left(x_{1}\right)$. That being the case, it follows from the argument used in the proof of Theorem 2, that the set $S=\left\{v_{k}^{\left(m_{1}\right)}\left(x_{1}\right) \mid \nabla(x)\right.$ 1s a solution of (1.1), $v_{\hat{k}}^{(1-1)}(\alpha)=q_{\hat{k}}^{(1-1)}(\alpha), 1 \leq 1 \leq n, v_{k}^{(1)}\left(x_{j}\right)=y_{k}^{(1)}\left(x_{j}\right), 0 \leq 1 \leq m_{j}-1,1 \leq j \leq q-1$, $\left.v_{k}^{(1)}\left(x_{q}\right)=y_{k}^{(1)}\left(x_{q}\right), 0 \leq 1 \leq m_{q}-2\right\}$ is an open interval. 
If for each $s \in S$, we let $\nabla(x, 8)$ denote the corresponding solution of (1.1), then there are distinct $s, s^{\prime} \varepsilon S$ such that $Y(x)=V(x, s)$ and $W(x)=V\left(x, 8^{\prime}\right)$. From the connectedness of $S$ and Theorem 3 , we conclude that there exists an $\overline{8} \varepsilon S$ which is between 8 and $s^{\prime}$ such that, for the kth component,

$$
0=v_{k}^{\left(m_{q}-1\right)}\left(x_{q}, 8\right)-v_{k}^{\left(m_{q}-1\right)}\left(x_{q^{\prime}}, 8^{\prime}\right)=\left(8-8^{\prime}\right) \frac{\partial v_{k}}{\partial 8}\left(m_{q}, \bar{s}^{-1)}\right. \text {. }
$$

If we set $Z(x, \bar{s})=\frac{\partial \nabla}{\partial s}(x, \bar{s})$, then $Z(x, \bar{s})$ is the solution of (1.2) along $V(x, \bar{s})$ and satisfies $z_{\hat{k}}^{(1-1)}(\alpha, \overline{8})=0,1 \leq 1 \leq n, z_{k}^{(1)}\left(x_{f}, \overline{8}\right)=0,0 \leq 1 \leq m_{f}-1$, $1 \leq f \leq q-1, z_{k}^{(1)}\left(x_{q}, \bar{s}\right)=0,0 \leq 1 \leq m_{q}-2$, and $z_{k}^{\left(m_{1}\right)}\left(x_{1},-s\right)=1$. But we also have above that $z_{k}^{\left(m_{q}-1\right)}\left(x_{q}, s\right)=0$, whlch contradicts the disconfugacy of $(1,2)$ on $(t, \sigma)$. Thus, our assumption is false and $\eta_{1}^{k}(t)=\operatorname{lnf}_{Y_{0}(x)}\left\{\eta_{1}^{k}\left(t ; Y_{0}(x)\right)\right\}$.

4. RIGHT DISPOCALITY AND INTERVALS OF RIGHT DISPOCALITY.

In this section we present analogues of the results of section 3 in terms of what we call k-component right disfocal1ty. Much of our notation is that used by Muldowney [10].

DEPINITIONS. Let $\tau=\left(t_{1}, \ldots, t_{n}\right)$ be an $n$-tuple of points from $(a, b)$. We say that a function $y(x)$ has $n$ zeros at $\tau$ provided $y\left(t_{1}\right)=0$, $1 \leq 1 \leq n$, and $y^{(f)}\left(t_{1}\right)=0,0 \leq j \leq m-1$, if $t_{1}$ occurs $m$ times in $\tau$. A partition $\left(\tau_{1} ; \ldots ; \tau_{\ell}\right)$ of $\tau$ 1s obtalned by inserting $\ell-1$ semicolons. Let $m_{1}=\left|\tau_{1}\right|$ be the number of components of $\tau_{1}$. We say that

$\left(\tau_{1} ; \ldots ; \tau_{\ell}\right)$ Is an 1ncreasing partition of $\tau$ provided $t_{1} \leq t_{2} \leq \ldots \leq t_{n}$, and if $t=t_{1}$, $s \in \tau_{f}$ with $1<f$, then elther $t<s$ or $t=s$ and $1+m \leq 1$, where $m$ is the mulliplicity of $t$ in $\tau_{1}$.

We say the system $(1.1)$ is $k$-component right $\left(m_{1} ; \ldots ; m_{l}\right)$ disfocal on $(a, b), 0 \leq m_{f} \leq n-j+1$, If given solutions $Y(x), Z(x)$ of $(1.1)$ such that their difference $W(x) \equiv Y(x)-Z(x)$ satisfies $w_{\hat{k}}^{(1-1)}(\alpha)=0,1 \leq 1 \leq n$, some $\alpha \varepsilon(a, b)$, and $w_{k}^{(f-1)}(x)$ has $m_{j}$ zeros at $\tau_{f}, 1 \leq f \leq \ell$, where $\left(\tau_{l} ; \ldots ; \tau_{\ell}\right) \quad 18$ an increasing partition of $n$ points in $(a, b)$ with $m_{f}=\left|\tau_{f}\right|$, then $1 t$ follows that $w_{k}(x) \equiv 0$.

For a sequence of integers $\left\{n_{f}\right\}_{j=1}^{l}$ satisfying

$$
n=n_{1}>n_{2}>\ldots>n_{\ell} \geq 1 \text {, }
$$

let $\left\{m_{f}\right\}_{j=1}^{\ell}$ be a sequence of nonnegative integers satisfying $m_{1}+\ldots+m_{\ell}=n, m_{2}+\ldots+m_{\ell} \leq n_{2}, \ldots, m_{\ell-1}+m_{\ell} \leq n_{\ell-1}, m_{\ell} \leq n_{\ell} \cdot(4.2)$ For a sequence $\left\{n_{f}\right\}_{f=1}^{\infty}$ satisfying $(4.1)$, define $B^{k}(t)=\sup \left(t_{1}>t \mid(1.1)\right.$ 1s $k$-component right $\left(m_{1} ; \ldots ; m_{\ell}\right)$ disfocal on $\left[t, t_{1}\right]$, for all sequences $\left\{m_{j}\right\}_{j=1}^{\ell}$ satisfying $(4.2)$ \}. Given a solution $Y(x)$ of $(1.1), B^{k}(t ; Y(x))$ is defined simflarly for the variational equation $(1.2)$ along $Y(x)$.

In much the same manner as Peterson [11] has proven for scalar equations, $\beta^{k}(t)$ can be characterized in terms of $\beta^{k}(t ; Y(x))$ as stated in the following theorem. 
THEOREM 6. Let $1 \leq k \leq m$ be given and let $c \varepsilon(a, b)$. Then, either (1) there is a solution $Y(x)$ of (1.1) such that the variational equation (1.2) along $Y(x)$ has a nontrivial solution $Z(x)$ satisfying the conditions

$$
\begin{array}{ll}
z_{\hat{k}}^{(1-1)}(\alpha)=0 & , 1 \leq 1 \leq n, \\
z_{k}^{(1-1)}(c)=0 & , 1 \leq 1 \leq n-1, \\
z_{k}^{(1-1)}(d)=0 & , n-j+1 \leq 1 \leq n+1,
\end{array}
$$

where $d=\beta^{k}(c ; Y(x)), \alpha \varepsilon[c, d]$, and $j$ satisfles $n_{n-j+2}<j \leq n_{n-j+1}$,

where $1 \leq n-j+1 \leq \ell,\left(n_{\ell+1}=0\right)$, or

$$
B^{k}(c)=\operatorname{lnf}_{Y(x)}\left\{B^{k}(c ; Y(x))\right\} .
$$

REPERENCES

1. PETERSON, A., Comparison theorems and existence theorems for ordinary differential equations, J. Math. Anal. Appl. 55 (1976), 773-784.

2. PETERSON, A., An expression for the first conjugate point for an nth order nonlinear differentlal equation, Proc. Amer. Math. Soc. 61 (1976), 300-304.

3. SPENCER, J., Relations between boundary value functions for a nonlinear differential equation and its varlational equations, Canad. Math. Bull. $\underline{18}(1975), 269-276$.

4. SUKUP, D., On the existence of solutions to multipoint boundary value problems, Rocky Mtn. J. Math. 6(1976), 357-375.

5. HENDERSON, J., Right focal point boundary value problems for ordinary differential equations and varlational equations, J. Math. Anal. Appl. 98 $(1984), 363-377$.

6. HENDERSON, J., Disconjugacy, disfocality, and differentiation with respect to boundary conditions, J. Math. Ana1. App1., to appear.

7. HENDERSON, J., K-point disconfugacy and disconjugacy for Iinear differential equations, J. Differential Eqs. 54(1984), 87-96.

8. MULDOWNEY, J., On disconfugacy and k-point disconjugacy for IInear ordinary differential operators, Proc. Amer. Math. Soc. 92 (1984), 27-30.

9. PETERSON, A., On a relation between a theorem of Hartman and a theorem of Sherman, Canad. Math. Bu11. 16 (1973), 275-281.

10. MULDOWNEY, J., On Invertibility of linear ordinary differential boundary value problems, SIAM. J. Math. Ana1. 12(1981), 368-384.

11. PETERSON, A., A disfocality function for a nonlinear ordinary differential equation, Rocky Mtn. J. Math. 12(1982), 741-752. 


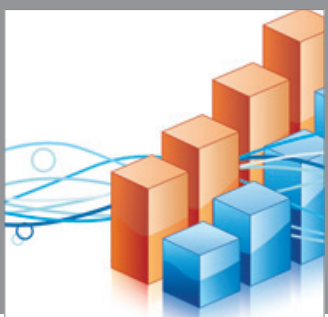

Advances in

Operations Research

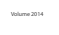

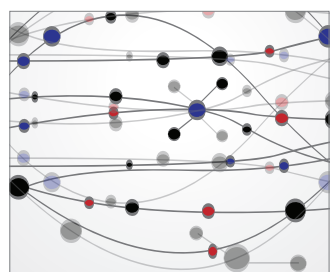

\section{The Scientific} World Journal
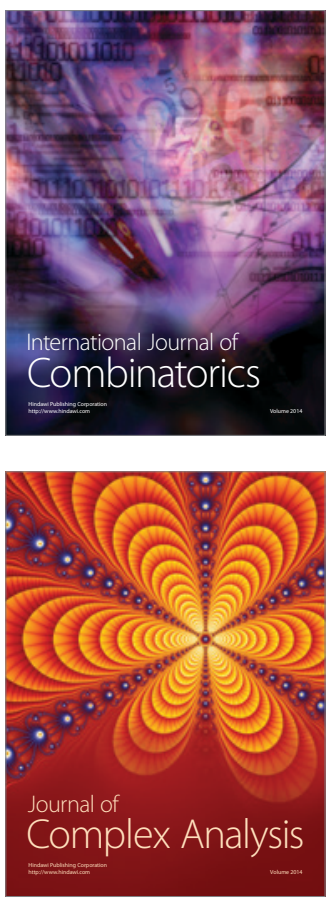

International Journal of

Mathematics and

Mathematical

Sciences
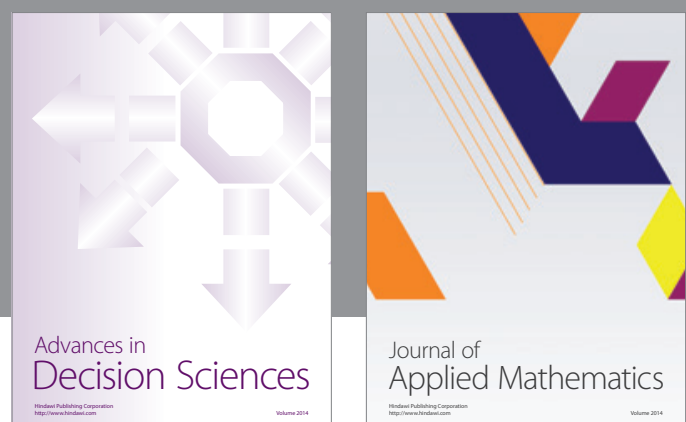

Journal of

Applied Mathematics
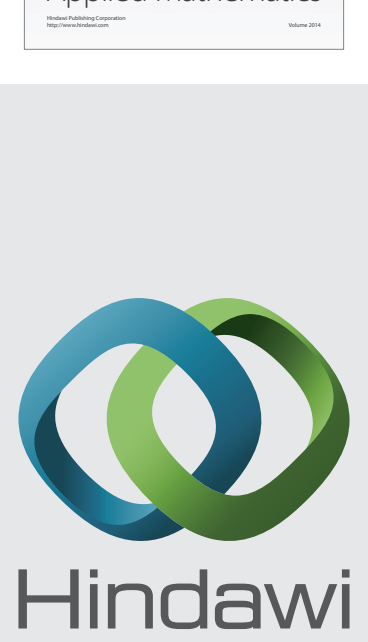

Submit your manuscripts at http://www.hindawi.com
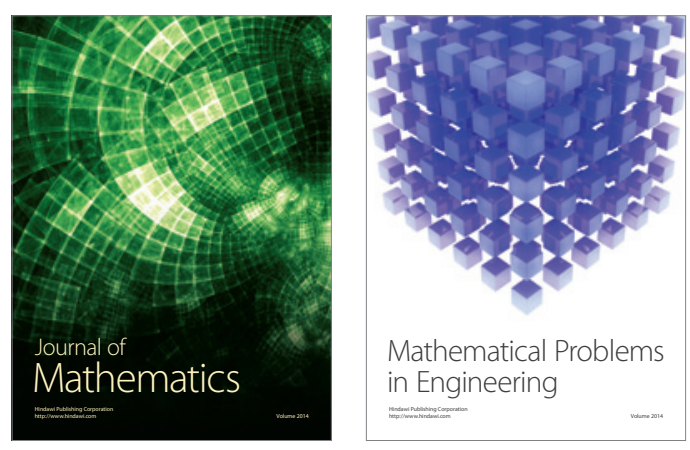

Mathematical Problems in Engineering
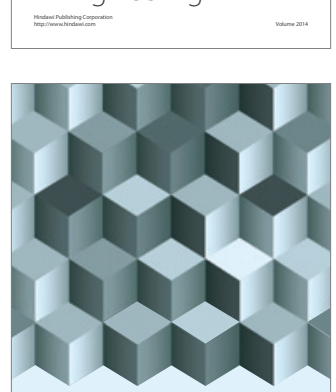

Journal of

Function Spaces
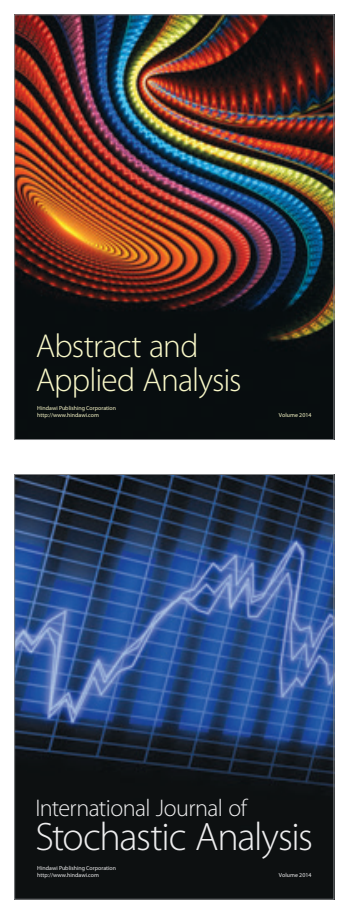

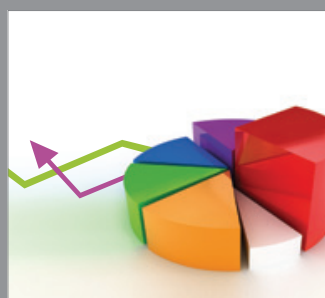

ournal of

Probability and Statistics

Promensencen
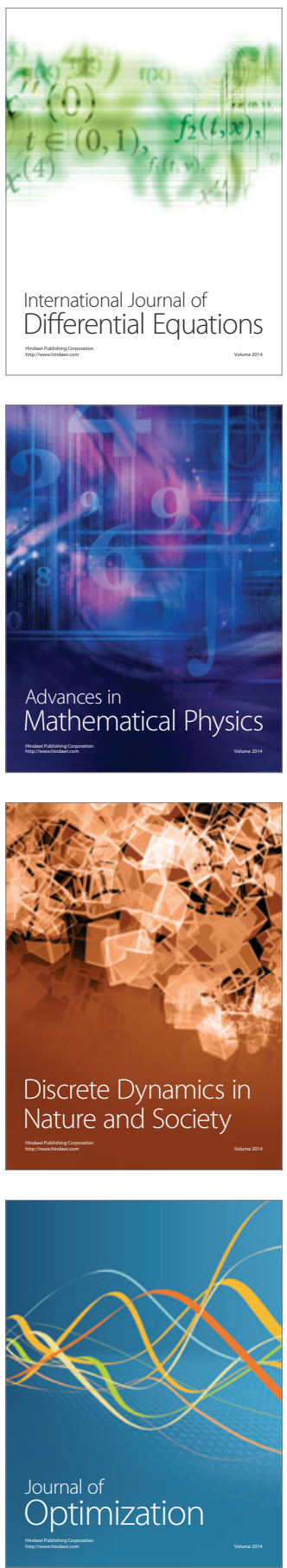